\title{
SURFACE WAVE DISPERSION MEASUREMENTS AND TOMOGRAPHY FROM AMBIENT SEISMIC NOISE CORRELATION IN CHINA
}

\author{
Xiaodong Song ${ }^{1}$, Xinlei Sun ${ }^{1}$, Sihua Zheng ${ }^{2}$, Zhen $\mathrm{Xu}^{1}$, Yingjie Yang $^{3}$, and Michael H. Ritzwoller ${ }^{3}$ \\ University of Illinois at Urbana-Champaign ${ }^{1}$, Institute of Earthquake Science, China Earthquake Administration ${ }^{2}$, \\ and University of Colorado at Boulder ${ }^{3}$ \\ Sponsored by Air Force Research Laboratory \\ Contract No. FA8718-07-C-0006
}

\begin{abstract}
We perform ambient noise tomography of China using the data from the China National Seismic Network and surrounding global and PASSCAL stations. The results so far are summarized below.

(1) Dispersion measurements and tomography. For most of the station pairs, we retrieve good Rayleigh waveforms from ambient noise correlations using 18-months of continuous data at all distance ranges across the entire region (over $5000 \mathrm{~km}$ ) and for periods from $70 \mathrm{~s}$ down to about $8 \mathrm{~s}$. We obtain Rayleigh wave group and phase velocity dispersion measurements using a frequency-time analysis method and invert for Rayleigh dispersion maps for periods from 8 to $60 \mathrm{~s}$. The dispersion maps correlate nicely with surface geology.
\end{abstract}

(2) Error estimates using bootstrap analysis. A major feature of the ambient noise method is that the whole process is completely repeatable with different time segments, which make it possible to evaluate the uncertainties. We adopt a bootstrap method to quantify the errors in the Rayleigh wave group velocity dispersion measurements and the tomographic maps. Most of the pairs show similar dispersion curves between different runs and small standard deviations, indicating good data quality and convergence of the Green function. Group velocity at long period end generally has a larger error, which is consistent with the notion that the long period needs longer time to converge. The best retrieved periods are from 10 to $30 \mathrm{~s}$ with the optimal period of around 15 to $20 \mathrm{~s}$. Pairs with large errors do not depend on the orientations of the paths or the locations of the stations. Rather, they are associated with a few stations with large average standard errors. The likely causes are missing data and poor instrumentation (or site conditions). Where ray coverage is good, there is only subtle difference in tomography maps between different runs, suggesting that our solution is very stable.

(3) 3D structure. We invert the Rayleigh group and phase dispersion maps for 3D shear-wave velocity structure. The 3D model shows some remarkable features, including slow sedimentary layers of all the major basins in China at the shallow depth, Moho depth variation, fast (strong) mid-lower crust and mantle lithosphere in major basins surrounding the Tibetan Plateau (TP) (Tarim, Ordos, and Sichuan). These strong blocks thus seem to play an important role in confining the deformation of the TP to be a triangular shape. The Moho change from plateau to Tarim is very sharp; the change to the Sichuan Basin is gentler but still steep. The India lithosphere seems to terminate around the Bangong Nujiang Suture as indicated by the fast-slow velocity contrast in the mantle lithosphere, but it seems to extend further north under E. Tibet. Widespread, prominent low-velocity zone is observed in mid-crust in the TP, providing strong support for the channel flow model. These slow velocities are generally connected and seem to reach to the surface near the margins of the TP, consistent with the thermalmechanical modeling of the ductile extrusion of the Greater Himalayan Sequence. 


\section{OBJECTIVES}

The overall objective of this project is to obtain surface wave dispersion measurements from ambient seismic noise correlations of the Chinese backbone stations (CNSN) and use these measurements to produce surface wave dispersion maps of China. More specifically, the objectives are: (1) to obtain dispersion measurements between CNSN stations; (2) to obtain dispersion measurements between CNSN and FDSN, regional networks, and temporary stations; (3) to obtain dispersion maps from these dispersion measurements.

\section{RESEARCH ACCOMPLISHED}

\section{Method and Data}

Recent theoretical and laboratory studies have shown that the Green functions of a structure can be obtained from the cross-correlation of diffuse wavefields (e.g., Lobkis and Weaver, 2001; see also review Campillo, 2006). The basic idea is that linear waves preserve, regardless of scattering, a residual coherence that can be stacked and amplified to extract coherent information between receivers. The idea has now found rapid applications in seismology. In particular, surface waves have been found to be most easily retrievable from the cross-correlations of seismic coda (Campillo and Paul, 2003) or ambient noise (Shapiro et al., 2005; Sabra et al., 2005) between two stations. Both Rayleigh waves and Love waves can be retrieved. The new type of data has rapidly been used for tomographic mapping at regional or local scales and on continental scales. These studies have focused on Rayleigh wave group velocity tomography from ambient noise. However, the method has been demonstrated to be applicable to Love waves and phase velocity measurements.

Ambient noise tomography (ANT) overcomes several important limitations of conventional methods based on earthquakes; i.e., uneven distribution of earthquake sources, uncertainty in earthquake location, and attenuation of short-period surface waves. Thus, the method is particularly useful for surface-wave path calibration and for tomographic mapping in aseismic regions especially at short periods (below $30 \mathrm{~s}$ ). In addition, the method can be applied any time without the need to wait for earthquakes and is completely repeatable, allowing quantification of errors.

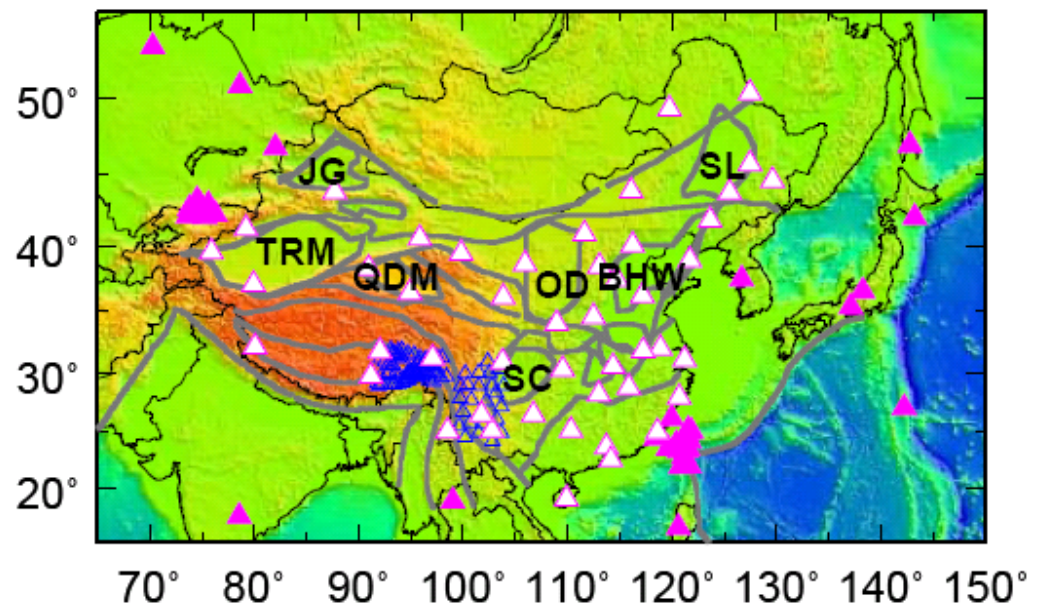

Figure 1. Distribution of seismic stations used in this study, including China National Seismic Network (CNSN) stations (solid white triangles), stations in the surrounding regions (solid pink triangles), and PASSCAL stations (open blue triangle). Plotted also are major tectonic boundaries and major basins. The basins that are labeled include: Tarim (TRM), Junggar (JG), Qaidam (QD), Sichuan (SC), Ordos (OD), Bohai Wan (BHW), and Songliao (SL) Basins.

Our main stations are from the China National Seismic Network (CNSN) (Figure 1). The CNSN is the national backbone network, established around 2000, with a relatively uniform distribution across the continental China. To increase data coverage, we also include a few stations in the surrounding regions. We focus on Rayleigh wave group velocities in this study. We use 18 months of continuous data from 47 CNSN stations and 32 international stations and 96 PASSCAL stations. All stations are broadband. The bandwidths of the CNSN stations are from $20 \mathrm{~Hz}$ to at least $120 \mathrm{~s}$. We use the data processing and imaging techniques described in great detail by Bensen et al. (2007). Below is a brief outline of our data procedure. First, we obtain the empirical Green function (EGF) from ambient noise cross-correlation. Continuous data are pre-processed before correlation and stacking, which includes clock 
synchronization, removal of instrument response, time-domain filtering, temporal normalization and spectral whitening. The purpose is to reduce the influence of earthquake signals and instrument irregularities and to enhance the strength and bandwidth of the ambient noise correlations. Second, if the signal-to-noise ratio (SNR) is sufficiently large, Rayleigh wave group speeds are measured using a frequency-time analysis (Ritzwoller and Levshin, 1998). Finally, the inter-station dispersion measurements are used to invert for the Rayleigh wave group velocity maps, in exactly the same way as earthquake-based measurements.

\section{Dispersion measurements and tomography}

For most of the station pairs, we are able to retrieve good Rayleigh wave signals from the ambient noise correlations (Zheng et al., 2008). Figure 2 shows typical examples of EGFs and group velocity measurements of Rayleigh waves retrieved from ambient noise correlations. The cross-correlations show strong arrivals at different settings (near the coast or well into the continental interior) and at both relatively low frequencies (20-50 s) and high frequencies (5$20 \mathrm{~s}$ ). The EGFs can be retrieved over the entire region (at distances of over $5000 \mathrm{~km}$ ) (Figure 2a).
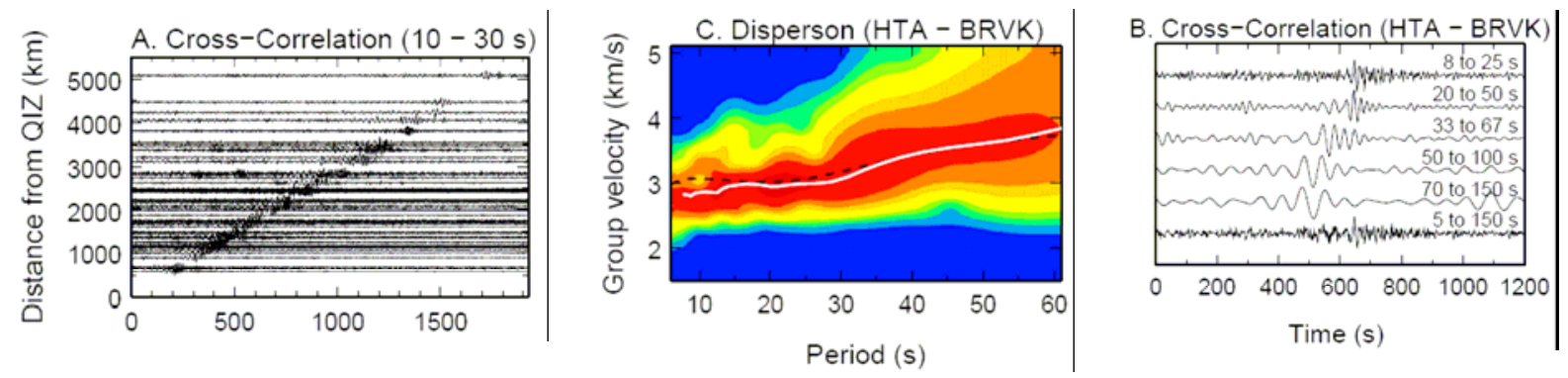

Figure 2. Example of Rayleigh wave EGFs and dispersion measurements obtained from ambient noise correlations. (a) Symmetric component of the correlations between station QIZ (in Hainan Province, China) and other stations. The traces are band-pass filtered at relatively short periods (10-30 s). (b) EGFs filtered in different frequency bands. Long-period surface waves are clearly faster than short-period ones. The path is between HTA (bordering Tarim in the south) and BRVK (Borovoye, Kazakhstan). (c) Frequency-time analysis (Ritzwoller and Levshin, 1998) used to retrieve Rayleigh wave group velocity dispersion curve (white) for the HTA-BRVK path. The black dashed curve is the prediction from the 3D global shear velocity model of Shapiro and Ritzwoller (2002), which is used for phasematched filtering in the data analysis and for comparison with measurements.
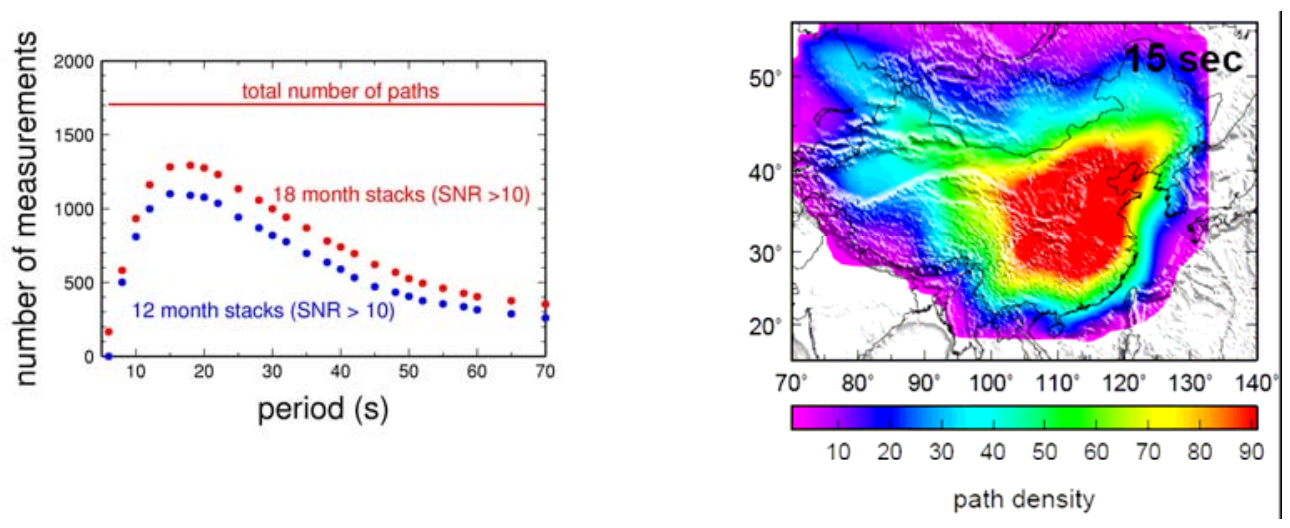

Figure 3. Distribution of dispersion measurements for different periods (left) ray density map for the period of $15 \mathrm{~s}$ (right). (Left) The total number of paths takes into account data availability for pairing up stations. The numbers of measurements obtained from 18-months of continuous (red dots) show about $20 \%$ of improvement over the numbers of measurements obtained using only 12-months of data (blue dots). (Right) The ray density is the number of rays inside 1 degree by 1 degree cell. The rays are station pairs for which dispersion measurements have been obtained. The ray coverage is best for periods 10 to $30 \mathrm{~s}$. Coverages for shorter or longer periods deteriorate, but the spatial coverage patterns remain similar. 
We measured group velocity dispersion curves (Figure 2c) for station pairs with Rayleigh wave SNR $>10$. The SNR is defined as the ratio of the peak amplitude of the Rayleigh wave to the root-mean-square value of the background. The measurement is very stable. Clear dispersion can be commonly observed directly from the EGFs (Figure 2b). We found that the group velocity measurements can extend to periods of $10 \mathrm{~s}$ or shorter even for station pairs that are separated over thousands of kilometers. The group velocities of the HTA-BRVK path (Figure $\mathbf{2 b}$,c), which samples the Tarim Basin, agree with a global 3D earthquake-based model (Shapiro and Ritzwoller, 2002) at longer periods but differ significantly at short periods (below $30 \mathrm{~s}$ ). The slow group velocities at short periods are caused by the thick sediments of the Tarim Basin (see discussion below).

We have obtained dispersion measurements with SNR $>10$ for periods $8 \mathrm{~s}$ to $70 \mathrm{~s}$ (Figure 3, left). The best observed frequency band is 10 to $30 \mathrm{~s}$ with over 1000 measurements at each period or a retrieval rate of 50 to $80 \%$ of all the possible pairs. The ray paths cover almost the entire Chinese continent (Figure 3, right). However, the coverage is much better in the eastern half of the country, because of the denser station distribution there than in the western part.

$10 \mathrm{~s}$

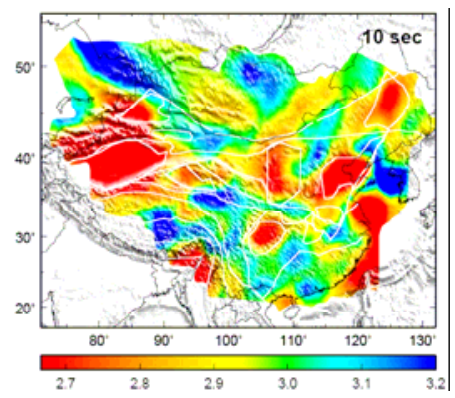

$40 \mathrm{~s}$

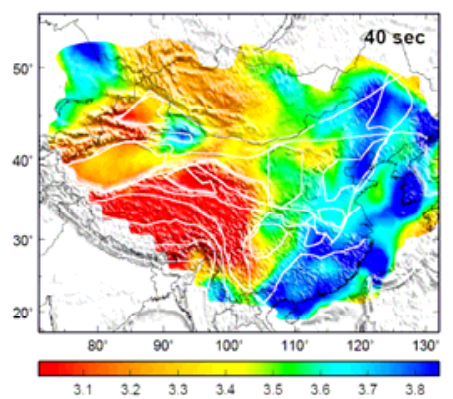

$20 \mathrm{~s}$

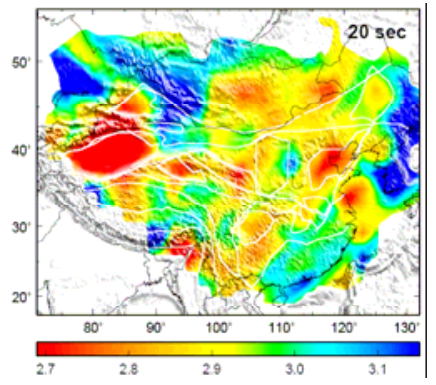

$50 \mathrm{~s}$

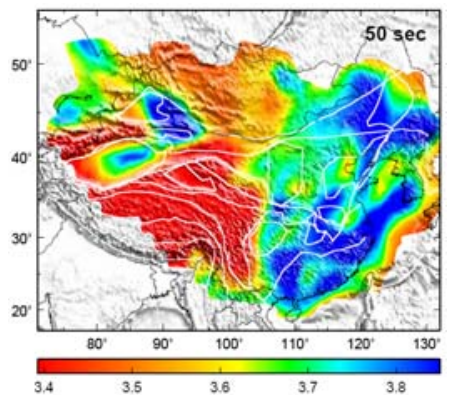

$30 \mathrm{~s}$

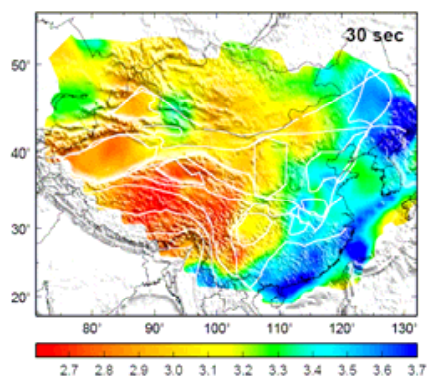

$60 \mathrm{~s}$

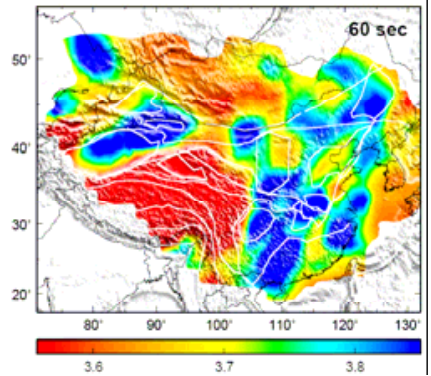

Figure 4. Rayleigh wave group velocities obtained in this study. Shown are the maps at periods 8, 15, 20, 25, 30, 40, 50, $60 \mathrm{~s}$, respectively. Plotted in the background are major block boundaries and basin outlines (Figure 1).

The ray coverage of our dispersion measurements is sufficient for us to invert for Rayleigh wave group velocity maps at periods from $8 \mathrm{~s}$ to $60 \mathrm{~s}$ (Figure 4). The results show remarkable features that correlate with large-scale geological structures of China. Major basins are well delineated with low velocities at short periods (8 to $20 \mathrm{~s}$ ), including Bohai-Wan Basin (North China Basin), Sichuan Basin, Qaidam Basin, and Tarim Basin. The stable Yangtz Craton also shows up well with high velocities. At longer periods (25-50 s), the group velocity maps display striking bimodal distribution with high velocity in the east and low velocity in the west, which corresponds very well with the thinner crust in the east and much thicker crust in the west (e.g. Liang et al., 2004). The NNE-SSW trending boundary between fast and slow velocities (around longitude $108^{\circ} \mathrm{E}$ ) coincides with the sharp topographic change and with the well-known Gravity Lineation.

A side-by-side comparison between a short period group velocity map and sediment thickness or between an intermediate period map and crustal thickness is presented in Figure 5. The correlations are quite striking if we compare the group velocities along a certain profile of interest (Figure 6). The selected profile along latitude of 
about $39^{\circ} \mathrm{N}$ passes through three major basins: Tarim, Ordos, and Bohai-Wan (Figure 1). The thick sediments in these basins (Laske and Masters, 1997) correlate well with slow velocities at periods from 10 to $20 \mathrm{~s}$ (Figure 6a).

The general trend of decreasing crustal thickness from west to east is well represented by increasing group velocities around $30 \mathrm{~s}$ (Figure 6b). However, the group velocity map displays more structure than the smooth crustal thickness curve from the global reference model (CRUST 2.0) (http://mahi.ucsd.edu/Gabi/rem.html), suggesting a more complex Moho. At period $50 \mathrm{~s}$, the trend is no longer observable as the surface waves sample deeper into the mantle.
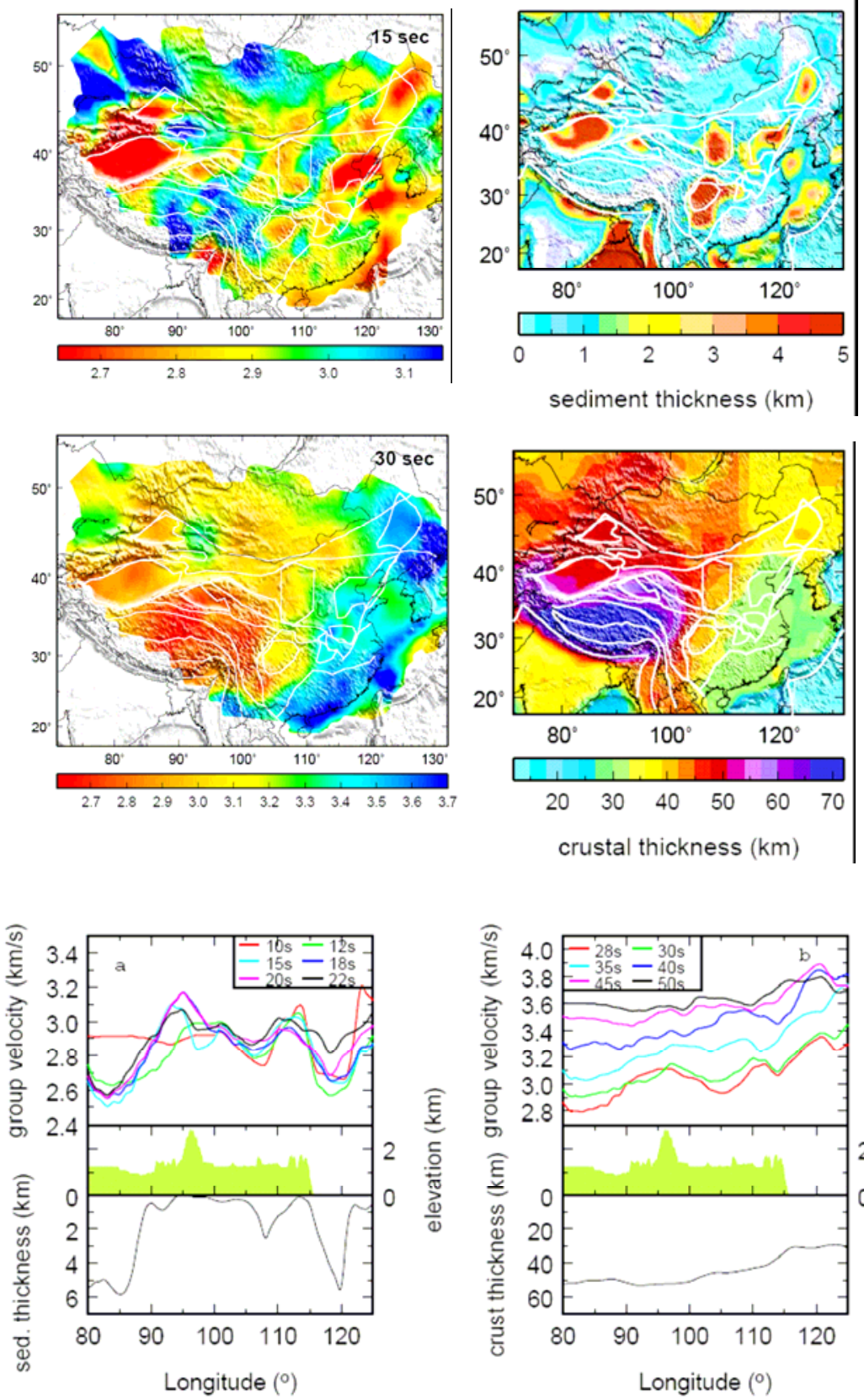

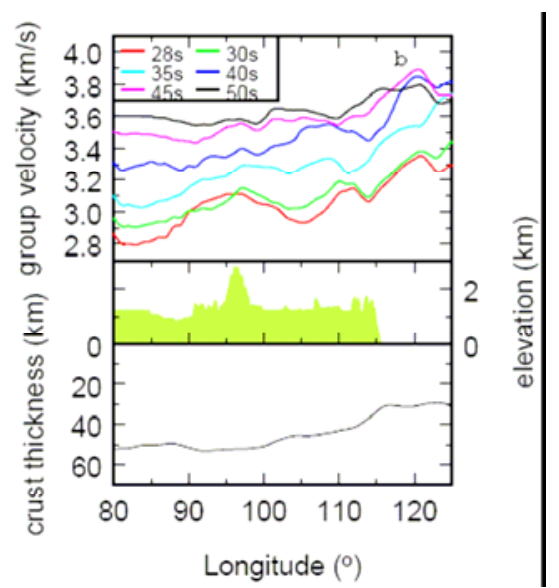

Figure 5. Comaprison of group velocities with and sediment thickness (top) and crustal thickness (bottom). (Top) The group velocity map (left) is for $15 \mathrm{~s}$ Rayleigh waves. The major basins (including Tarim, Junggar, Qadaim, Sichuan, Bohai Wan, Songliang, as well as the Southern North China and Jianghang basins in the eastcentral region) are well delineated by slow velocities. See Figure 1 for the location of the basins. (Bottom) The group velocity map is for $30 \mathrm{~s}$ Rayleigh waves. The major trend of crust thickening from the east to west (right) is well represented by the velocity decreases from east to west (left).

Figure 6. Rayleigh wave group velocities are compared with sediment thickness (a) and crustal thickness (b) along a selected path. The selected path is along the great-circle path between $\left(38^{\circ} \mathrm{N}, 80^{\circ} \mathrm{E}\right)$ and $\left(38^{\circ} \mathrm{N}, 125^{\circ} \mathrm{E}\right)$, passing through three major basins (Tarim, Ordos, and Bohai Wan) (Figure 1). Plotted at the top are velocities for shorter periods (a) and for longer periods (b), respectively. Plotted at the bottom are sediment thickness (a) and crustal thickness (b), respectively. In the middle are surface elevations. 


\section{Validation and error analyses of dispersion measurements and tomography}

Comparison of the tomographic maps with the geological features discussed above provides an important initial validation of the ambient noise tomography (ANT) methodology; i.e., the method provides models of group wave speeds that are consistent with well-known geological features and other geophysical observations. Furthermore, the complete repeatability of the ANT method makes it possible to validate directly the methodology and to evaluate the uncertainties of the dispersion measurements. Several methods have been proposed previously in this regard (see summary by Zheng et al., 2008). (1) Direct verification: Comparing the EGF with the surface wave generated by an earthquake along the same path. (2) Comparing the EGF obtained from ambient noise and that from seismic coda. (3) Temporal stability: Comparing the EGFs from the data observed at different time periods (e.g., different months). Furthermore, because the principal ambient noise sources are believed to come from the oceans, which are seasonal, the consistency of the correlations from different seasons gives a measure of the stability and error of the EGFs. (4) Spatial consistency: Comparing the EGFs for station-pairs along similar paths. The EGFs between a faraway station to two or more stations that are close to one another should be similar as the paths sample similar structure.

We have examined temporal and spatial consistency of our dispersion measurements and found that they are very consistent whenever the SNRs of the EGFs are high. Some examples are shown in Figure 7. The temporal comparisons include a station pair with an east-west path (BJT-BRVK) and another pair with a north-south path (XAN-CHTO) (Figure 7, top). We construct 23 dispersion curves using 12-months of data with different sliding windows or using 12-months of data over different seasons. For either pair, we find the standard deviation of these curves to be less than $2 \%$ for all periods and the standard deviation of the mean to be less than $0.5 \%$. The spatial comparisons include two pathways (Figure 7, bottom), from GOM to GZH/SZN and from WMQ to SSE/NJ2. The group velocities between WMQ and SSE are quite similar to those between WMQ and NJ2 at all the observed periods (10 to $60 \mathrm{~s}$ ). The group velocities between GOM-GZH and GOM-SZN are also similar at periods less than $40 \mathrm{~s}$. At periods greater than $40 \mathrm{~s}$, they are somewhat different but are within the uncertainties as indicted in the temporal plots (Figure 7, top).
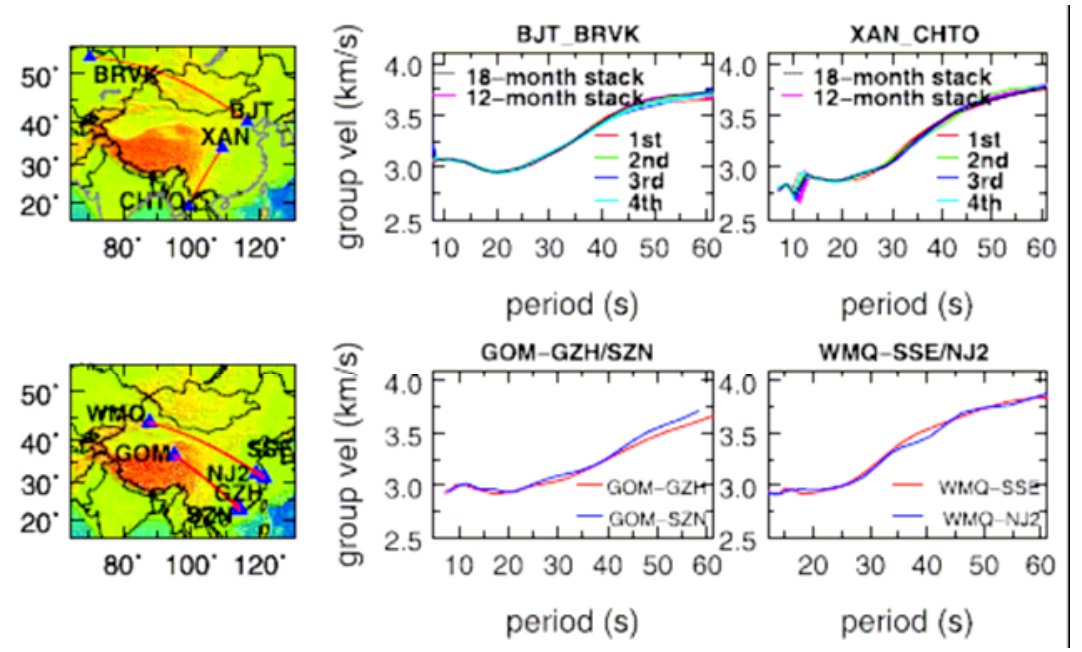

Figure 7. Temporal (top) and spatial (bottom) consistency of dispersion measurements. (Top) Dispersion curves from different time windows. We select two pairs, BJT-BRVK along an east-west path and XAN-CHTO along a north-south path. For either pair, we calculate two sets of EGFs. For each calculation of the EGF, a total of 12 months of data are used. The 18-month stack (including all the data we collected) is plotted for comparison. One set uses seasonal data (red, green, blue, and cyan), i.e., data from the same season over a period of 4 years. The other set uses 12-months of data with a sliding time window of 10 days (total of 19 curves, all in magenta). (Bottom) Dispersion curves between a far-way station and two close stations. We select two pathways, one from GOM to GZH/SZN (distance about $2400 \mathrm{~km}$ ) and the other from WMQ to SSE/NJ2 (distance about $3100 \mathrm{~km}$ ). The distance between GZH and SZN is about $133 \mathrm{~km}$, and that between SSE and NJ2 is $245 \mathrm{~km}$. 

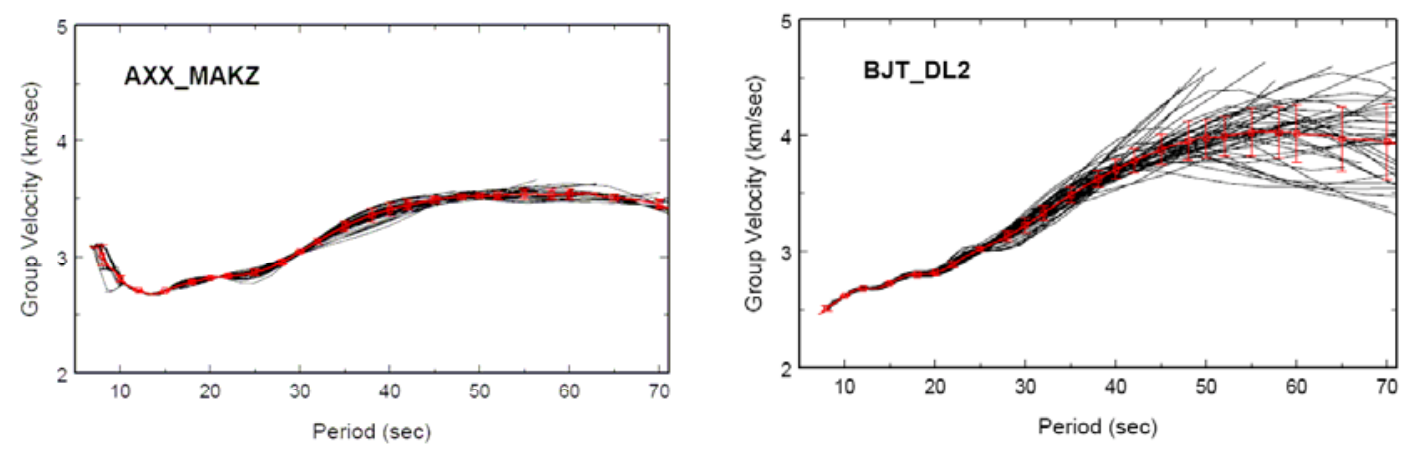

Figure 8. Examples of error estimates of dispersion curves from bootstrap with a good pair (left) and a bad pair (right). Black curves are dispersion measurements for all the 50 runs. Red curves are the mean values. Error bars at different periods indicate the standard deviations. For the good pair (AXX-MAKZ), dispersion curves for different runs are very close to each other. The standard deviations are small throughout the whole periods. For the bad pair (BJT-DL2), dispersion curves spread out at long periods. The large standard deviations at long periods indicate large errors in the measurements.
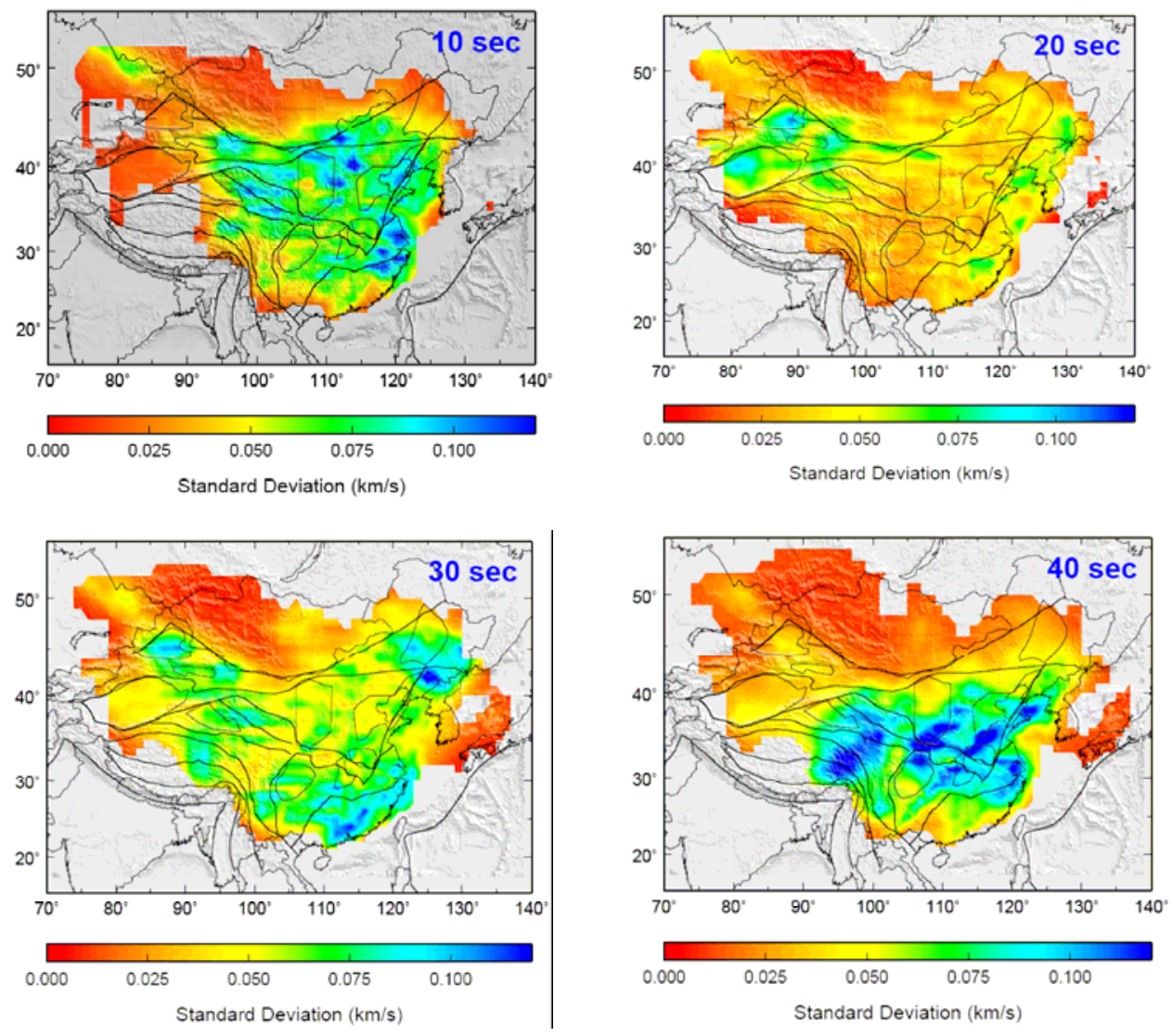

Figure 9. Maps showing standard deviations of Rayleigh group velocity dispersion models at periods of $10 \mathrm{~s}$ (a), 20 $\mathrm{s}(\mathrm{b}), 30 \mathrm{~s}(\mathrm{c})$, and $40 \mathrm{~s}$ (d). Color scales are the same in all the figures. Minimum standard deviation appears at about $20 \mathrm{~s}$. As the period increases, the error also increases. Red color at the border region is an artifact from smoothing constraint and regularization. In the central part of China with best ray coverage, the small standard deviations indicate the robustness of the tomography results. 
The retrieval of the surface wave EGF relies on the stacking of cross correlation of continuous data for long enough time series. The total length of data required for the Green function to converge is empirical and highly frequency dependent: generally the longer the period the longer the time is needed. Furthermore, the ambient noise source and station site conditions (including instrumentation stability) are uncertain. We have recently proposed a bootstrap method to quantify the errors in Rayleigh group velocity dispersion measurements and group velocity tomographic maps, based on the complete repeatability of the ambient noise correlation and tomography process (Xu et al., 2008).

Our bootstrap analysis follows the following steps. 1) We obtain the EGF using one month of data for each of the 18 months. 2) We select 18 random months among the months that we have data. The selection process is a random sampling with replacement as in any boostrap methods. We then obtain the statck EGF using the EGFs of these months for each and every station pair. Using these stacked GFs, we measure dispersion curves for all station pairs and construct tomographic maps as usual. 3) We repeat the step 2 for 50 times. 4) We obtain the mean and the standard deviation of the dispersion curve for each station pair from the 50 dispersion curves obtained in step 3 . We regard the mean and the standard deviation as the group velocity estimate and the associated error. 5) Similarly, we obtain the standard errors of our tomographic models using the models obtained from the 50 iterations described in steps 2 and 3.

Figure 8 shows two examples of error estimates of the dispersion curves using the boostrap method. Most pairs show similar dispersion curves between different runs and small standard deviation (generally less than $0.1 \mathrm{~km} / \mathrm{s}$ ), indicating good data quality and convergence of the Green function. Group velocity at a long period generally has a larger error, which is consistent with the notion that the long period needs longer time to converge. There is only subtle difference in tomography maps between different runs, suggesting that our solution is very stable (Figure 9). Standard deviation in the region with good ray coverage is small (generally less than $0.1 \mathrm{~km} / \mathrm{s}$ ), indicating a stable and reliable solution in well-sampled regions. The Rayleigh waves are best retrieved from 10 to $30 \mathrm{~s}$ with the best periods around 15 to $20 \mathrm{~s}$. A pitfall of the model error estimates is that the standard deviations in the regions with poor ray coverage (at the margins) are also small, due to regularization in the tomographic inversion process. Our tomographic inversion includes regularization using a prior model. Thus the inversions for the poorly sampled regions from different runs all converge to the prior model, giving an artifact of small errors.

We found that the pairs with large variations do not have a preferred orientation or a particular location (Figure 10, left). Rather, these pairs are generally associated with a few stations with large standard deviations. We derive average standard deviation of the surface wave velocity for each station at each period by averaging over all the pairs associated with that station. Figure 10 (right) shows average standard deviations of dispersion measurements for individual stations over all periods. A few stations have standard deviations of greater than $0.19 \mathrm{~km} / \mathrm{s}$. The exercises provide a way to identify and sort out good and poor quality stations efficiently.

\section{D shear-wave structure}

We combine our Rayleigh wave group and phase velocity maps (8-60 s) with longer-period dispersion maps from global earthquake-based measurements (Ritzwoller and Levshin, 1998). We then invert the dispersion maps for 3D S structures using programs by Bob Herrmann of St. Louis U. Preliminary results show clearly some remarkable features for continental China and in particular the Tibetan Plateau (TP) (Figure 11), including slow sedimentary layers of all the major basins at the shallow depth, striking east-west contrasts in Moho depth variation and lithosphere thickness, fast (strong) mid-lower crust and mantle lithosphere in major basins surrounding the TP (Tarim, Ordos, and Sichuan) (in contrast, Qaidam Basin does not have such a "deep root"). These strong blocks thus seem to play an important role in confining the deformation of the TP to be a triangular shape. The Moho changes from plateau to Tarim and Sichuan Basins are quite sharp. The India lithosphere seems to terminate around the Bangong Nujiang Suture (BNS) as indicated by the fast-slow velocity contrast in the mantle lithosphere, consistent with many previous studies, but it seems to extend further north under E. Tibet. In northwest TP, slow anomalies extend from crust to great depth $(200 \mathrm{~km})$. 


\section{Period 18 (sec)}

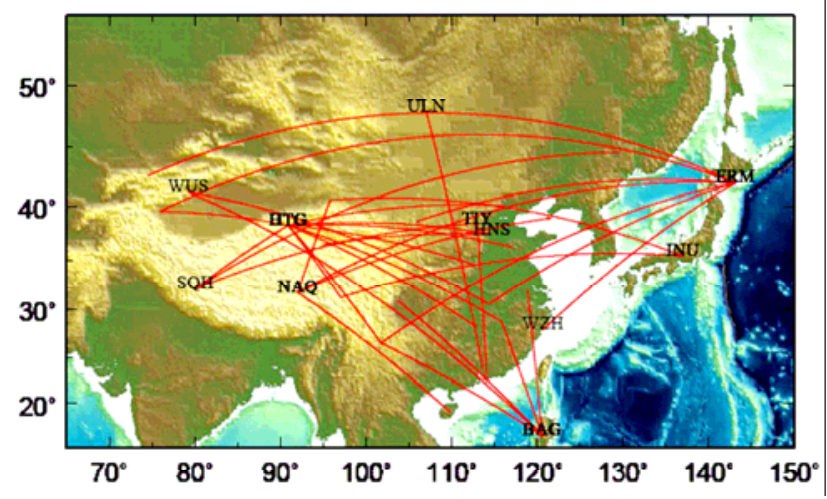

Average Standard Diviation

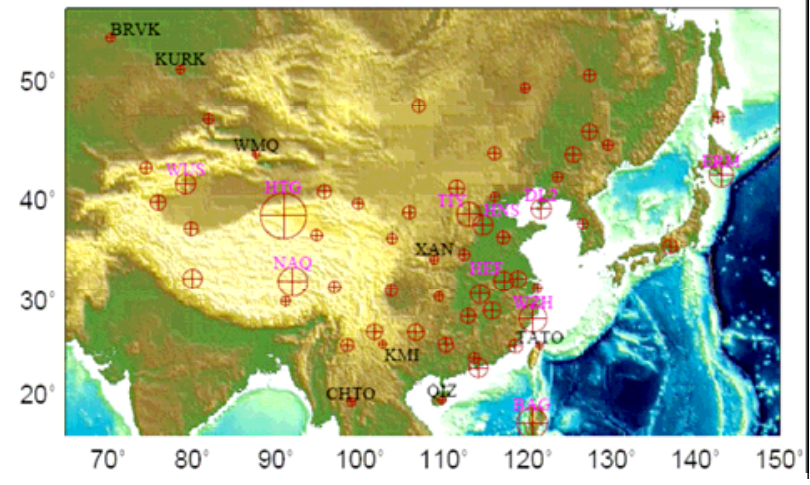

Figure 10. (Left) Station pairs with standard deviations larger than $0.5 \mathrm{~km} / \mathrm{sec}$. The bad pairs are generally associated with few a stations with large average standard deviations in the dispersion measurements (BAG, NAQ, HTG, ERM). (Right) Average standard deviations of dispersion measurements for individual stations plotted at the station sites. The size of the red cross or the open circle represent the size of average standard deviation for the station. The larger the symbol size is, the larger the error is. Stations with average standard deviations smaller than $0.09 \mathrm{~km} / \mathrm{s}$ are labeled in black color, while stations with standard deviations larger than $0.19 \mathrm{~km} / \mathrm{s}$ are labeled in pink color.
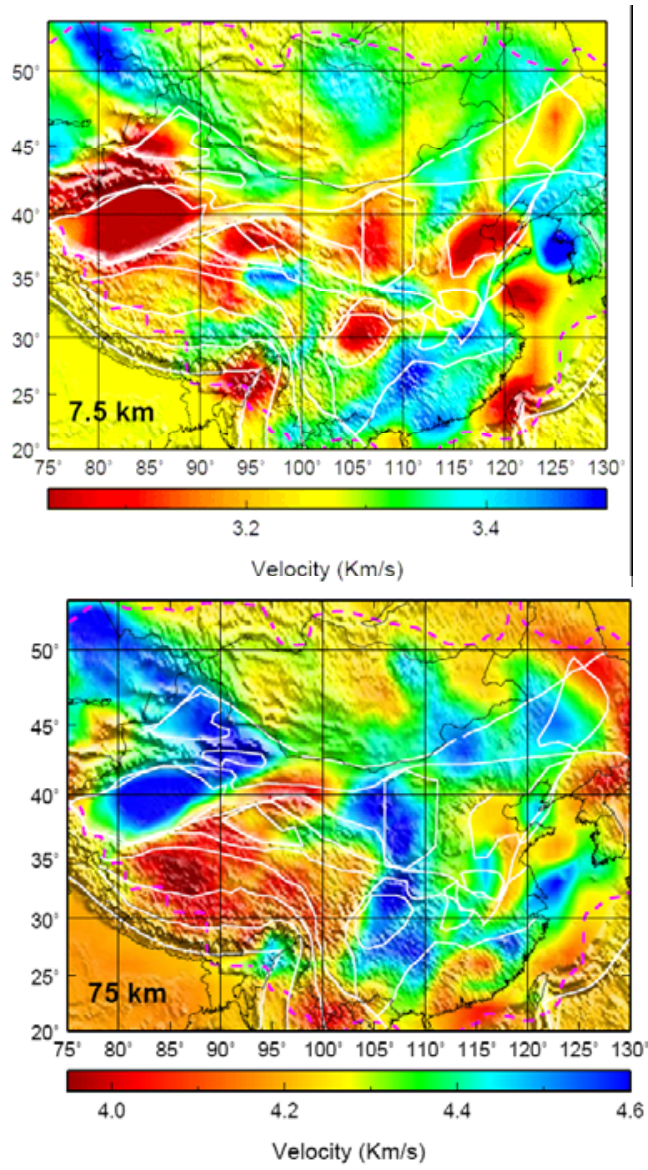
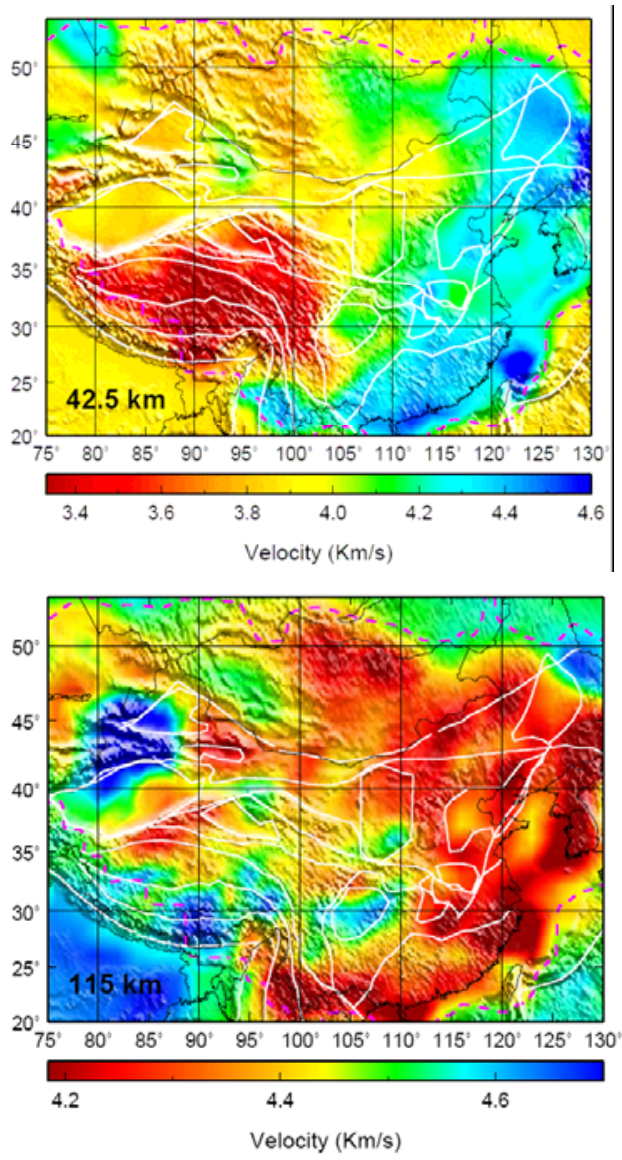

Figure 11. Shear-wave velocity at $7.5 \mathrm{~km}$ (upper left), $42.5 \mathrm{~km}$ (upper right), $75 \mathrm{~km}$ (lower left), and $115 \mathrm{~km}$ (lower right). 
Extremely slow $\mathrm{S}$ velocities mark the mid-crust in much of the central, eastern, and southeastern TP. These slow velocities are widespread and often (but not always) connected in a laminar form. They seem to reach to the surface at certain localities in western, southern, northern, and southeastern margins. The mid/lower crust low velocity zone provides support for the channel flow model that has been proposed for the outward growth and uplift of the TP (e.g. Clark and Royden, 2000) and for the extrusion of crustal materials to the surface (e.g., Beaumont et al., 2001).

\section{CONCLUSIONS AND RECOMMENDATIONS}

We have obtained Rayleigh dispersion curves for periods of 8 to $70 \mathrm{~s}$ for $~ 10,000$ new inter-station paths from ambient noise correlation using correlations of 18 months of continuous data from CNSN, international permanent, and PASSCAL temporary stations. The best observed frequency band is 10 to $30 \mathrm{~s}$ with a retrieval rate of 50 to $80 \%$ of the station-pairs. We constructed new tomographic maps of China for periods from 8 to $60 \mathrm{~s}$, which correlate with surface geology. Temporal and spatial analyses suggest that the dispersion measurements and tompography are robust. We proposed a bootstrap method, which allowed us to quantify the errors of each of our dispersion curves and the errors of the dispersion maps. For best periods (10-30 s) and most regions, the Rayleigh waves have standard errors of less than $0.1 \mathrm{~km} / \mathrm{s}$. We have inverted the dispersion maps for 3D S model (down to about $200 \mathrm{~km}$ ). The models show remarkable details of the crust and uppermost mantle, including major blocks, rapid changes in Moho, $\mathrm{mid} /$ lower crust velocities in TP, and variations in lithosphere velocities.

We will add more PASSCAL stations, refine our error estimates of dispersions and tomographic maps, and conduct resolution tests of $3 \mathrm{D}$ structure.

\section{REFERENCES}

Beaumont C, Jamieson RA, Nguyen MH, et al., Himalayan tectonics explained by extrusion of a low-viscosity crustal channel coupled to focused surface denudation, Nature, 414, 738-742, 2001.

Benson, G., M. Ritzwoller, M. Barmin et al. (2007), Processing seismic ambient noise data to obtain reliable broadband surface wave dispersion measurements, Geophys. J. Int., 169(3), 1239-1260.

Campillo, M. Phase and correlation in random seismic fields and the reconstruction of the Green function, Pure Appl. Geophys., 163, 475-502, 2006.

Campillo M. and A. Paul, Long-range correlations in the diffuse seismic coda, Science, 299, 547-549, 2003.

Clark, M.K., and L.H. Royden, Topographic ooze: Building the eastern margin of Tibet by lower crustal flow, Gelogy, 28, 703-706, 2000.

Liang C., X.D. Song, and J.L. Huang, Tomographic Inversion of Pn Travel-Times in China, J. Geophys. Res., 109, B11304, doi. 10.1029/2003JB002789, 2004.

Lobkis, O.I., and R.L. Weaver, On the emergence of the Greens function in the correlations of a diffuse field, $J$. Acoust. Soc. Am., 110, 3011-3017, 2001.

Ritzwoller, M.H. and A.L. Levshin, Eurasian surface wave tomography: Group velocities, J. Geophys. Res., 103, 4839-4878, 1998.

Sabra, K.G., P. Gerstoft, P. Roux, W.A. Kuperman, and M.C. Fehler, Surface wave tomography from microseisms in Southern California, Geophys. Res. Lett., 2005.

Shapiro, N.M. M. Campillo, L. Stehly, and M.H. Ritzwoller, High resolution surface wave tomography from ambient seismic noise, Science, 307(5715), 1615-1618, 2005.

Xu, Z., X.D. Song, S.H. Zheng, M.H. Ritzwoller, Bootstrap analysis on surface wave dispersion and tomography derived from ambient noise cross-correlation, IRIS Workshop, Skamania Lodge, Stevenson, WA, 2008.

Zheng, S.H., X.L. Sun, X.D. Song, Y.J. Yang, M.H. Ritzwoller, Surface wave tomography of China from ambient seismic noise correlation, Geochem. Geophys. Geosyst., 9, Q05020, doi:10.1029/2008GC001981, 2008. 